\section{Role of calcitonin in management of musculoskeletal pain}

\author{
Lars Arendt-Nielsen, ${ }^{1,2}$ Hans Christian \\ Hoeck, ${ }^{2,4}$ Morten A. Karsdal, ${ }^{3}$ \\ Claus Christiansen ${ }^{2,3,4}$ \\ ${ }^{1}$ Center for Sensory-Motor Interaction, \\ Department of Health Science and \\ Technology, Aalborg University, Aalborg; \\ ${ }^{2}$ C4Pain, Aalborg; ${ }^{3}$ Nordic Bioscience \\ A/S, Herlev; ${ }^{4}$ CCBR-Synarc, Aalborg/ \\ Ballerup, Denmark
}

\section{Abstract}

Calcitonin was discovered more than 40 years ago and the scientific community continues to debate the primary and secondary pharmacological actions of calcitonin. Presently calcitonin is accepted by agencies only for treatment of osteoporosis, but many studies have indicated an effect on pain in many different experimental settings both pre-clinically and clinically. The effects of calcitonin on clinical pain conditions have received increasing attention in the past decades, although a consensus on mode of action and potential indications still has to be reached. Several key advances in the pain field may enable a deeper understanding of the putative analgesic effects of calcitonin. Most studies have focused on the effect of calcitonin on musculoskeletal pain problems. Ample lines of independent evidence suggest that calcitonin exerts putative analgesic effects. Well-designed clinical trials, particularly in the field of musculoskeletal pain, are needed to validate fragmented evidence of analgesic actions. This in combination with advanced mechanism-based pain assessment tools can provide new insight into the role of calcitonin, alone or in combination with other compounds, in management of pain.

\section{Introduction}

Non-malignant musculoskeletal pain is the most common clinical symptom that causes patients to seek medical attention and is a major cause of disability in the world. Musculoskeletal pain can arise from a variety of common conditions including osteoarthritis, rheumatoid arthritis, osteoporosis, surgery, low back pain, and bone fracture.

A major problem in designing new therapies to treat musculoskeletal pain is that the underlying mechanisms driving musculoskeletal pain are not understood fully, although recent years of intensive focus have highlighted the role of central sensitization as a key mechanism. ${ }^{1}$ Further achievements in acquiring knowledge are hampered by the fact that animal models of musculoskeletal pain do not mirror the human condition closely, which again slows down the development of novel therapies to treat musculoskeletal pain. A mechanismbased understanding of the factors involved in generating musculoskeletal pain has gained acceptance in recent years and will develop the area further. ${ }^{2}$ Developing both an understanding of the mechanisms that drive persistent musculoskeletal pain and novel mechanismbased therapies to treat these unique pain states would address a major unmet clinical need and have significant clinical, economic, and social benefits. Calcitonin (CT) may have a mechanism of action that interferes with key aspects of chronic musculoskeletal pain.

The aim of this review is to highlight the possible intrinsic analgesic properties of CT, with special focus on the challenges in the musculoskeletal system. In this respect osteoporosis and osteoarthritis are of particular interest as they are very frequent, painful musculoskeletal pain conditions; therefore they have been dealt with separately in the following discussion.

In order to locate the corpus of studies that directly compared calcitonin and/or pain we conducted a literature search of the Medline (from 1965) databases to June 2009, entering the search terms: calcitonin, pain, musculoskeletal, joint, bone, spine, nociception, clinical trial, and management. We obtained studies that may have been missed in our previous searches by conducting a manual review of journals that historically publish on calcitonin and pain (i.e. J Bone Miner Metab, Bone, Joint Bone Spine, Osteoarthritis Cartilage, Osteoporos Int, Pain, Clin J Pain).

Relevant articles were entered into Science Citation Index to retrieve reports that have cited these articles, and conference proceedings and textbooks were searched manually. As 35 references were allowed for this review the selection of studies to be cited was based on the authors' best judgments of which were most representative.

\section{Calcitonin, pain, and osteoporosis}

CT was discovered more than 40 years ago and possesses potent anti-resorptive effects that are mediated by direct binding of CT to its receptor on the osteoclasts. CT is a peptide hormone produced by parafollicular cells (Ccells) in the thyroid gland, and which is secreted in response to excess calcium in the serum. Various sources of CT exist, of which salmon
Correspondence: Lars Arendt-Nielsen, Center for Sensory-Motor Interaction (SMI), Department of Health Science and Technology, Aalborg University, Fredrik Bajers Vej 7, D3, DK-9220 Aalborg E, Denmark. E-mail: LAN@hst.aau.dk

Key words: calcitonin, pain, arthrosis, osteoporosis.

Received for publication: 3 June 2009.

Revision received: 25 November 2009.

Accepted for publication: 26 November 2009.

Contributions: LAN and MK wrote the initial draft of the paper, and $\mathrm{HCH}$ and $\mathrm{CC}$ provided valuable critical input.

Conflict of interest: $\mathrm{MK}$ and $\mathrm{CC}$ are involved in clinical trials on the effect of calcitonin on osteoporosis, osteoarthritis, and pain.

This work is licensed under a Creative Commons Attribution 3.0 License (by-nc 3.0).

(O) Copyright L. Arendt-Nielsen et al., 2009

Licensee PAGEPress, Italy

Rheumatology Reports 2009; 1:e12

doi:10.4081/rr.2009.e12

from the teleost family is the most potent.

CT is approved for the treatment of osteoporosis and other diseases involving accelerated bone turnover. ${ }^{3}$ Until now CT treatment has been limited to either subcutaneous or intranasal administration. However, recently a novel oral formulation has received increased attention. ${ }^{3}$ Adverse effects of CT therapy such as sedation, nausea, skin flushing, and diarrhea may reflect increased serotonergic activity. Salmon calcitonin (sCT) is safe and effective in the treatment of osteoporosis, reducing the risk of new vertebral fractures in postmenopausal women, and improves the clinical outcome after joint arthroplasty. Pain is associated often with fractures, ${ }^{4}$ and thus pain management is a central although secondary problem associated with bone and joint damage. ${ }^{4}$

The role of sCT as an analgesic in relation to managing pain in osteoporosis has been addressed less as the researchers interested in osteoporosis are not concerned with pain and vice versa.

\section{Calcitonin, pain, and osteoarthritis}

Osteoarthritis (OA) is the most common form of arthritis. ${ }^{5}$ A hallmark of the disease is progressive degeneration of articular cartilage and subsequent joint space narrowing. In the majority of patients the etiology of $\mathrm{OA}$ is not known. Among the known risk factors of $\mathrm{OA}$ are age, significant traumata, obesity, altered gait, altered biomechanics (e.g. varus or vagus 
deformity), and excessive loading.

Experimental and clinical observations suggest that the structural integrity of articular cartilage is dependent on normal subchondral bone turnover, intact chondrocyte function, and ordinary biomechanical stresses. An increasing line of evidence suggests that there are strong interrelationships between the subchondral bone and the articular cartilage. As bone and cartilage are closely interrelated, interventions affecting pain related to bone turnover may influence 0A-relevant pain as well. Pain is a central part of the clinical presentation of $\mathrm{OA}^{6}{ }^{6}$ and may drive patients to seek consultation for the later diagnosis of $\mathrm{OA}$. Many and diverse structural features of the joint have been implicated in the pain associated with $\mathrm{OA}$, including but not limited to the presence of osteophytes in the patellofemoral compartment, focal or diffuse cartilaginous abnormalities, subchondral cysts, bone marrow edema, subluxation of the meniscus, meniscal tears, and Baker cysts. ${ }^{6}$ However, pain perception is highly individual and very few features correlate to pain robustly. ${ }^{6}$

Normal adult articular cartilage is thought to be avascular and aneural. However, very recently sympathetic and sensory nerves were identified within vascular channels in the articular cartilage in both mild and severe OA. Perivascular and free nerve fibers, and nerve trunks were observed within the subchondral bone marrow and the marrow cavities of osteophytes. Nerve endings localized in damaged human articular cartilage suggest that vascularization and the associated innervation of articular cartilage may contribute to tibiofemoral pain in OA across a wide range of structural disease severity. The implications of these findings are that the musculoskeletal pain associated with 0 A may result from a combination of the previously accepted central and bone-derived effects.

Current recommendations for symptomatic treatment of $\mathrm{OA}$ list paracetamol ( $4 \mathrm{~g}$ per day) as the first-line analgesic. However, in a recent survey of patients with OA only a third of the patients found paracetamol effective. Over $60 \%$ of the patients responded better to nonsteroidal anti-inflammatory drugs (NSAIDs). ${ }^{7}$ Current recommendations suggest that long-term use of NSAIDs for management of OA pain should be avoided. It is obvious that current OA management regimes do not focus at all on possible peripheral and central manifestations of this chronic pain condition. One reason for this is the lack of understanding of the underlying causes of pain in $\mathrm{OA}$.

A systematic review and meta-analysis of randomized placebo controlled trials to estimate the analgesic efficacy of NSAIDs, including selective cyclo-oxygenase-2 inhibitors (coxibs), in patients with OA of the knee has been published recently. ${ }^{8}$ The study concluded that
NSAIDs reduced short-term pain in OA of the knee slightly more than placebo did. However, the analysis did not support long-term use of NSAIDs for this condition as serious adverse effects can be associated with oral NSAIDs. Another recent meta-analysis study evaluated randomized controlled trials of short duration only (less than four weeks) assessing the efficacy of topical NSAIDs in 0A. ${ }^{9}$ After two weeks there was no evidence of efficacy superior to placebo. No trial data support the long-term use of topical NSAIDs in OA.

The lack of efficacy of current medications in $\mathrm{OA}$ indicates that the mechanisms involved are not responsive to the therapies, and alternative approaches may be needed. Peripheral and central pain sensitization in $\mathrm{OA}$ seems to be a very prominent feature. New knowledge of the involved basic mechanisms and better methods to assess muscle pain in the clinic are needed to revise and optimize treatment regimes. Increased muscle sensitivity is manifested as: (1) pain evoked by a normally non-nociceptive stimulus (allodynia), (2) increased pain intensity evoked by nociceptive stimuli (hyperalgesia), or (3) increased referred pain areas with associated somatosensory changes. Some manifestations of sensitization have been put forward, such as expanded referred muscle pain areas in $0 A$ patients ${ }^{10}$ and from animal experiments showing extra-segmental spread of sensitization. ${ }^{11}$ An important part of the pain manifestations (e.g. tenderness and referred pain) related to chronic musculoskeletal disorders may be because of peripheral and central sensitization, which play a role in the transition from acute to chronic pain.

Many clinical studies report increased sensitivity to painful stimuli of deep tissues in both localized and referred pain areas in musculoskeletal pain conditions. ${ }^{12}$ Referred pain often is clinically manifested as the spread of pain from the site of injury/damage, and has been known and described for more than a century, resulting in its extensive use as a diagnostic tool in the clinic. Pain manifestation in OA as an important clinical sign of changes in the central nervous system often is hampered by the phenomenon of spreading, which influences the effect of traditional analgesics used as they do not influence the central manifestations.

Currently, several ongoing clinical trials are exploring the role of CT in 0A-related pain, and the data will be interesting to follow because CT, as described in the following section, may interact with some of the central manifestations related the central sensitization.

\section{Clinical evidence of calcitonin in pain control}

The analgesic activity of sCT (subcutaneous or intranasal) has been known for many years and shown in several controlled prospective double-blind studies, ${ }^{13}$ although the mechanism of action is not known. Direct epidural or subarachnoidal injection of CT also has shown an analgesic effect. Besides pain management in osteoporosis, ${ }^{14,15}$ CT has shown an analgesic action in painful conditions such as phantom limb pain, ${ }^{16}$ diabetic neuropathy, ${ }^{17}$ complex regional pain syndrome, ${ }^{18}$ adhesive capsulitis, ${ }^{19}$ rheumatoid arthritis, ${ }^{20}$ vertebral crush fractures, ${ }^{14}$ spondylitis, tumor metastasis, ${ }^{21}$ cancer pain ${ }^{22}$ migraine ${ }^{23}$ Paget's disease of bone, ${ }^{24}$ as well as postoperative pain. ${ }^{25}$ Interestingly and which may hint toward the mode of action, epidural CT in combination with local anesthetic produces an analgesic effect similar to that of fentanyl and with stable hemodynamic results. ${ }^{13}$ It also eliminates postoperative hyperglycemia indicating that CT is a suitable alternative for the treatment of acute postoperative pain. ${ }^{13}$ With specific regard to musculoskeletal pain and osteoporosis, patients receiving $200 \mathrm{IU}$ of sCT nasal spray per day consumed significantly less analgesic per day than patients in the placebo group. ${ }^{26}$ Furthermore, data from crush fractures show greater pain relief (within 7 days) with nasal sCT spray (200 IU) than with intramuscular injection. The analgesic effect is associated with an elevation of the circulating levels of $\beta$-endorphin, which seems to be higher with the nasal form.

\section{Possible mechanisms underly- ing calcitonin analgesia}

The acute effect of sCT in animals is in contrast to the clinical experience where the onset of analgesia is slow, most likely because CT slowly accumulates in the brain inducing analgesia when sufficient receptors are occupied. Exogenous sCT has to cross the blood-brain barrier to cause analgesia, and hence doses administered centrally may act faster than higher doses administered more peripherally.

Mammalian CT appears less potent and less rapid than CT from other species, and the nasal routes, appears better than the subcutaneous or intramuscular route, at least on the release of $\beta$-endorphin, which is rapidly increased. ${ }^{27}$ Clinically the analgesic effect seems to last long (weeks) possibly owing to accumulation in brain tissue and strong binding to the receptors.

The CT family of peptides comprises calcitonin, amylin, two calcitonin gene-related peptides (CGRP $\alpha$ and $\beta$ ), and adrenomedullin. CT binds to a seven-membrane-spanning array-G protein-coupled receptor (similar in structure to an opioid receptor) with cyclic-AMP and calcium acting as secondary messengers. Calcitonin and calcitonin gene-related peptide (involved in pain transmission, neurogenic inflammation, 
and migraine related mechanisms) share the same gene on chromosome 11 in humans, and both peptides exhibit similar structural and receptor binding properties. CT receptors are present on serotonergic neurons in the thalamus, periaqueductal gray matter, nucleus gigantocellularis, and raphe nucleus, which are all regions involved in the processing of pain. This interaction with the serotonergic pathways is particularly important as the descending pain inhibitory pathways seem to be affected by chronic musculoskeletal pain, and lesioning the serotonergic pathways or the use of antagonist drugs decreased the analgesic potency of CT in animals. $^{28}$

Percile $^{29}$ suggested that the CT inhibition of prostaglandin E2 synthesis was an explanation of its analgesic effect. However, it is clear that other pathways and systems are involved, such as the cholinergic or serotonergic systems $\mathrm{Ca}^{2+}$ fluxes, protein phosphorylation, cyclooxygenase inhibition, histamine-induced vascular leakage, and proopiomelanocortin ( $\beta$-endorphin precursor) production. The role of the in-creased plasma $\beta$-endorphin after CT administration has been debated heavily. It has been suggested that $\beta$-endorphin decreases the synthesis of prostaglandins or other humoral factors, modulating pain via a central mechanism, or perhaps has an effect on local pain mediators through CT-binding sites in the periphery. Several studies indicate that the inhibitory de-scending serotonergic systems may be invol-ved, through 5-HT1A and 5-HT3 receptors, in the production of an antinociceptive effect by centrally injected $\mathrm{CT}^{28}$

The role of CT in the central nervous system has received increased attention as animal studies have indicated that the antinociception in the CNS, for example in the pons, is suggesting pain modulation via descending systems. This descending pain control is supported further by the $\beta$-endorphin release, which increases the activity in the nucleus raphe magnus, consequently activating the serotonergic descending pathway and inhibiting substance $P$ release. In patients with bone metastases $\beta$-endorphin levels are found to rise with an increasing dose of CT. The CNS effects are supported further by the finding of CT receptors in the hypothalamic region, brain regions (e.g. medial pontine reticular formation and the rostrocaudal axis of the periaqueductal gray matter) involved in nociceptive processing, and in the dorsal horn of the spinal cord. Apart from the possible role of the endogenous released opioid $\beta$-endorphin from the pituitary gland, it has been ruled out in some studies that $\beta$-opioid receptors are involved in mediating CT analgesia, as naloxone did not antagonize sCT analgesia. A more recent study demonstrated that CT interacts with the $\mu-, \delta$, and $\kappa$-opioid receptors with the most prominent effect for the $\delta$ - and $\kappa$-agonists. ${ }^{30}$

\section{Experimental pain models and calcitonin}

\section{Animal experimental studies}

Electrical stimulation of the dental pulp and the hot-plate test have been used as an experimental model to assess analgesic efficacy of CNS-administered sCT. For some reasons the effects assessed by the hot-plate test seem less consistent. Approximately 80 minutes after central administration of $\mathrm{CT}$ in rabbits the nociceptive reaction to dental electrical stimulation is blocked maximally. In the formalin models of persistent pain CT has shown an effect on both the early and late formalin responses. There seems to be little evidence for any peripheral CT actions on nociceptors but modulating the synthesis of the inflammation mediator and calcium flux may affect peripheral targets.

\section{Clinical setting}

One human experimental pain study using CT on healthy volunteers has been performed. ${ }^{31}$ Welzel and Welzel found that subcutaneously administered CT increased the pain threshold to electrical stimulation of the dental pulp in a dose-dependent manner. The experimental study, in contrast to clinical experience, showed a rapid analgesic onset (approximately 80 minutes) and a short duration (up to 180 minutes). The effect of CT (IV 200 IE) in patients with chronic phantom pain did not show any modulation of pain thresholds to electrical (temporal summation), thermal, and pressure stimulation, ${ }^{32}$ indicating that the pain parameters evaluated may not represent the possible pain mechanisms affected by CT. Experimental pain models, such as pressure algometry, have been used in clinical studies. In osteoporotic patients the group treated with sCT had significantly lower pain scores to standardized pressure-applied pain (corresponding to an increase in pain threshold) at each assessment than the group receiving placebo. ${ }^{26}$ The pressure-pain threshold over a vertebral fracture was found to be elevated significantly after sCT in patients with a recent osteoporotic spinal fracture. ${ }^{26} \mathrm{CT}$ was found to affect cartilagespecific collagen type II fragments, as a marker of cartilage degradation, significantly. ${ }^{3}$ Lastly preliminary evidence of oral CT in patients with OA showed a significant effect on pain and joint function. ${ }^{33}$

Future pain studies may focus more on the characterization, the fundamental and intrinsic effect, and the mode of action of sCT analgesia. These studies in contrast to earlier studies may take advantage of new methods in pain research, focusing on central sensitization and impaired descending inhibition, and thus prove informative from a mechanistic point of view.

\section{Compounds interacting with the analgesic effect of calcitonin}

Antidepressants (noradrenaline and/or serotonin reuptake inhibitors) are used as a treatment modality for neuropathic pain, for example. Most of them act by selectively blocking reuptake. The interaction between $\mathrm{CT}$ and the serotonin system has been confirmed, as blocking the serotonin receptor blocks the effect of CT. The synergetic analgesic effect between CT and serotonin reuptake inhibitor antidepressants has been shown, suggesting joint administration of an antidepressant and CT in pain management. Furthermore, the interaction between CT and the descending serotonergic pain modulatory pathways may suggest clinical applications in patients with impaired descending pain control, as this is found in many patients with chronic pain. Inefficient descending noxious inhibitory control (DNIC) mechanisms have been reported in patients experiencing chronic pain with temporomandibular disorders, chronic low back pain, irritable bowel syndrome, fibromyalgia, complex regional pain, painful $\mathrm{OA}$, and chronic tension-type headaches. Interestingly, the patients in the OA study first experienced a deficient DNIC-like pain inhibition but showed normal inhibition after surgery when they were in a pain-free state, suggesting that the chronic pain maintained the DNIC dysfunction. Recently Yarnitsky et al. ${ }^{34}$ found that the potency of DNIC predicted the development of chronic pain after thoracotomy, indicating that DNIC impairment can be used as a prognostic factor for the development of chronic pain, whereas patients expressing efficient DNIC at the pain-free stage before surgery are less likely to develop chronic postoperative pain and, if having pain, will have less intense pain than those with less efficient DNIC. In a continuation study, ${ }^{35}$ it was shown that preoperative DNIC explained $24.6 \%$ of the variability in chronic postoperative pain intensity, further suggesting that drugs like sCT, interacting with the descending modulation, may have new applications in patients with chronic pain. sCT has been shown to interact with the opioid receptors with the most prominent agonistic effect on the $\delta$ - and $\kappa$-receptors. ${ }^{30}$ This may hint at some interesting aspects of CT's mode of action as $\delta$-receptors are expressed densely in periosteal and $\kappa$-receptors in visceral structures. This may explain the beneficial clinical effect on pain in relation to bone metastasis and after intra-abdominal and extra-abdominal surgery.

There is one human experimental clinical study on chronic phantom limb pain ${ }^{32}$ where CT, CT and ketamine in combination, or ketamine alone was given. Together with the clinical pain parameters some experimental pain 
measures (pain thresholds to electrical [temporal summation], thermal, and pressure stimulation) were recorded. The study showed no beneficial effect on either clinical or experimental measures of combining an NMDAreceptor antagonist (ketamine) with CT. The interaction between CT and vitamin D is evident in osteoporosis for example, and recent studies have indicated a possible role of vitamin D in pain. Patients with OA with low vitamin D levels have increased pain, with the most pronounced effect in females. Therefore, it may be of interest to look into the combined effect of CT and vitamin D on pain, in particular musculoskeletal pain, as vitamin D is a wellrecognized player in the continuous balance of turnover of the musculoskeletal system.

\section{Future perspectives}

With the recent clinical complications and withdrawal from the market of various COX-2 inhibitors there is a medical need for alternative treatment regimes in the field of musculoskeletal pain, and it seems that CT alone or in combination with other compounds may be an interesting alternative for some conditions. New and more advanced studies are needed to investigate if CT is interacting with some of the key mechanisms involved in chronic musculoskeletal pain.

\section{References}

1. Arendt-Nielsen L, Graven-Nielsen T. Deep tissue hyperalgesia. J Musculoskel Pain 2002;10:97-119.

2. Arendt-Nielsen L, Graven-Nielsen T. Translational aspects of musculoskeletal pain: from animals to patients. In: Fundamentals of Musculoskeletal Pain. Graven-Nielsen T, Arendt-Nielsen L, Mense S, editors. Seattle: IASP Press 2008;347-66.

3. Bagger YZ, Tanko LB, Alexandersen P, et al. Oral salmon calcitonin induced suppression of urinary collagen type II degradation in postmenopausal women: A new potential treatment of osteoarthritis. Bone 2005;37: 425-30.

4. Blau LA, Hoehns JD. Analgesic efficacy of calcitonin for vertebral fracture pain. Ann Pharmacother 2003;37:564-70.

5. Abramson SB, Attur M, Yazici Y. Prospects for disease modification in osteoarthritis. Nat Clin Pract Rheumatol 2006;2:304-12.

6. Ragab Y, Emad Y, Abou-Zeid A. Bone marrow edema syndromes of the hip: MRI features in different hip disorders. Clin Rheumatol 2008;27:475-82.

7. Wolfe F, Zhao S, Lane N. Preference for non- steroidal anti-inflammatory drugs over acetaminophen by rheumatic disease patients: a survey of 1,799 patients with osteoarthritis, rheumatoid arthritis, and fibromyalgia. Arthritis Rheum 2000;43:378-85.

8. Bjordal JM, Ljunggren AE, Klovning A, et al. Non-steroidal anti-inflammatory drugs, including cyclo-oxygenase-2 inhibitors, in osteoarthritic knee pain: meta-analysis of randomised placebo controlled trials. $\mathrm{Br}$ Med J 2004;329:1317.

9. Lin J, Zhang W, Jones A, et al. Efficacy of topical non-steroidal anti-inflammatory drugs in the treatment of osteoarthritis: meta-analysis of randomised controlled trials. Br Med J 2004;329:324.

10. Bajaj P, Bajaj P, Graven-Nielsen T, et al. Osteoarthritis and its association with muscle hyperalgesia: an experimental controlled study. Pain 2001;93:107-14.

11. Mense S. Nociception from skeletal muscle in relation to clinical muscle pain. Pain 1993;54:241-89.

12. Lautenbacher S, Rollman GB, McCain GA. Multi-method assessment of experimental and clinical pain in patients with fibro-myalgia. Pain 1994;59:45-53.

13. Gabopoulou Z, Vadalouca A, Velmachou K, et al. Epidural calcitonin: does it provide better postoperative analgesia? An analysis of the haemodynamic, endocrine, and nociceptive responses of salmon calcitonin and opioids in epidural anesthesia for hip arthroplasty surgery. Pain Pract 2002;2:326-31.

14. Lyritis GP, Paspati I, Karachalios T, et al. Pain relief from nasal salmon calcitonin in osteoporotic vertebral crush fractures. A double blind, placebo-controlled clinical study. Acta Orthop Scand Suppl 1997;275:112-4.

15. Knopp JA, Diner BM, Blitz M, et al. Calcitonin for treating acute pain of osteoporotic vertebral compression fractures: a systematic review of randomized, controlled trials. Osteoporos Int 2005;16:1281-90.

16. Jaeger H, Maier C. Calcitonin in phantom limb pain: a double-blind study. Pain 1992;48:21-7.

17. Zieleniewski W. Calcitonin nasal spray for painful diabetic neuropathy. Lancet 1990; 336:449.

18. Appelboom T. Calcitonin in reflex sympathetic dystrophy syndrome and other painful conditions. Bone 2002;30:S84-6.

19. Waldburger M, Meier JL, Gobelet C. The frozen shoulder: diagnosis and treatment. Prospective study of 50 cases of adhesive capsulitis. Clin Rheumatol 1992;11:364-8.

20. Sileghem A, Geusens P, Dequeker J. Intranasal calcitonin for the prevention of bone erosion and bone loss in rheumatoid arthritis. Ann Rheum Dis 1992;51:761-4.

21. Martinez-Zapata MJ, Roque M, AlonsoCoello P, et al. Calcitonin for metastatic bone pain. Cochrane Database Sys Rev
2006;3:CD003223.

22. Braga PC, Dal Sasso M, Bernini A, et al. Antinociceptive activity of salmon calcitonin: electrophysiological correlates in a rat chronic pain model. Neurosci Lett 1993;151:85-8.

23. Gennari C, Chierichetti MS, Gonnelli S, et al. Migraine prophylaxis with salmon calcitonin: a cross-over double-blind, placebocontrolled study. Headache 1986;26:13-6.

24. Altman RD, Collins-Yudiskas B. Synthetic human calcitonin in refractory Paget's disease of bone. Arch Intern Med 1987;147:1305-8.

25. Miralles FS, Lopez-Soriano F, Puig MM, et al. Postoperative analgesia induced by subarachnoid lidocaine plus calcitonin. Anesth Analg 1987;66:615-8.

26. Gennari C. Analgesic effect of calcitonin in osteoporosis. Bone 2002;30:S67-70.

27. Ofluoglu D, Akyuz G, Unay 0, et al. The effect of calcitonin on beta-endorphin levels in postmenopausal osteoporotic patients with back pain. Clin Rheumatol 2007;26:44-9.

28. Ito A, Kumamoto E, Takeda M, et al. Mechanisms for ovariectomy-induced hyperalgesia and its relief by calcitonin: participation of 5-HT1A-like receptor on Cafferent terminals in substantia gelatinosa of the rat spinal cord. J Neurosci 2000;20:6302-8.

29. Pecile A. Calcitonin and relief of pain. Bone Miner 1992;16:187-9.

30. Goicoechea C, Ormazabal MJ, Alfaro MJ, et al. Effect of salmon-calcitonin on the analgesic effect of selective mu, delta and kappa opioid agonists in mice. Neurosci Lett 1999;262:25-8.

31. Welzel K, Welzel D. Increase of pain threshold by calcitonin during electrical stimulation of the dental pulp. Investigations in healthy volunteers with regard to plasma concentration. Schmerz 1989;3:118-32.

32. Eichenberger U, Neff F, Sveticic G, et al. Chronic phantom limb pain: the effects of calcitonin, ketamine, and their combination on pain and sensory thresholds. Anesth Analg 2008;106:1265-73.

33. Manicourt DH, Azria M, Mindeholm L, et al. Oral salmon calcitonin reduces Lequesne's algofunctional index scores and decreases urinary and serum levels of biomarkers of joint metabolism in knee osteoarthritis. Arthritis Rheum 2006;54:3205-11.

34. Yarnitsky D, Crispel Y, Eisenberg E, et al. Prediction of chronic post-operative pain: pre-operative DNIC testing identifies patients at risk. Pain 2008;138:22-8.

35. Granot M, Weissman-Fogel I, Crispel Y, et al. Determinants of endogenous analgesia magnitude in a diffuse noxious inhibitory control (DNIC) paradigm: do conditioning stimulus painfulness, gender and personality variables matter? Pain 2008;136:142-9. 